\title{
TIFA Promotes Cell Survival and Migration in Lung Adenocarcinoma
}

\author{
Wanfu Men Wenya Li Jungang Zhao Yu Li \\ Department of Thoracic Surgery, The First Affiliated Hospital of China Medical University, Shenyang, \\ China
}

\section{Key Words}

TIFA $•$ Lung adenocarcinoma $•$ Proliferation $•$ Apoptosis

\begin{abstract}
Background/Aims: TNF- $\alpha$ receptor-associated factor (TRAF)-interacting protein with a forkhead-associated (FHA) domain (TIFA) may mediate the impact of TRAF on the development of lung cancer. The current study was conducted to investigate the expression of TIFA in lung adenocarcinoma and its potential role in the regulation of cancer cell proliferation and migration, and its influence on patient survival. Methods: Specimens of lung adenocarcinoma tissues and their adjacent normal lung tissues were obtained from 116 patients who underwent surgical resection of lung cancer. The expression of TIFA in the lung tissues was examined by immunohistochemistry, immunoblotting, and real-time RT-PCR in four different lung cancer cell lines and one normal bronchial epithelial cell line (BEAS-2B). TIFA was silenced by RNAi technique, and cell proliferation was then assessed by the CCK8 method. Furthermore, cell migration was determined by wound-healing trans-well and wound-healing migration assays. Additionally, cell-cycle arrest and apoptosis were assessed by flow cytometry analysis. Results: TIFA was positively detected in 63 (54.3\%) out of 116 lung adenocarcinoma specimens, which was significantly higher than the respective rate established in normal tissues adjacent to the tumor $(30.1 \%, p<0.05)$. The overall survival rate was significantly lower in the patients with positive TIFA expression than that in the patients with negative TIFA expression $(p<0.05)$. TIFA was also highly expressed in the lung cancer cell lines (H1299, H1975, and HCC827) tested. It is noteworthy that siRNA suppressed the expression of TIFA, which contributed to the attenuation of cell proliferation and migration, but promoted cell-cycle arrest and apoptosis. Furthermore, the silencing of TIFA caused upregulation of p53, p21, and cleaved-caspase-3, but downregulation of $\mathrm{BCl}-2$, cyclin $\mathrm{D} 1$, and CDK4, as well as phosphorylation of IKKB, IKB, and p65. Conclusions: TIFA may serve as a biomarker in the prediction of lung adenocarcinoma. Furthermore, TIFA may modulate lung cancer cell survival and proliferation through regulating the synthesis of apoptosis-associated proteins.
\end{abstract}




\section{Cellular Physiology Cell Physiol Biochem 2018;47:2097-2108 and Biochemistry Published \begin{tabular}{l|l} 
DOI: 10.1159/000491478 & $\begin{array}{l}\text { C } 2018 \text { The Author(s). Published by S. Karger AG, Basel } \\
\text { www.karger.com/cpb }\end{array}$
\end{tabular} \\ Men et al.: TIFA in Lung Adenocarcinoma}

\section{Introduction}

Lung cancer is the leading cause of cancer-associated death in the world [1]. Non-small cell lung cancer (NSCLC) cases constitute the majority (up to 80\%) of all lung cancer cases, with a continuously increasing prevalence of adenocarcinoma over the recent years $[2,3]$.

The tumor microenvironment is a milieu of pro-inflammatory responses, mediated by a variety of inflammatory cytokines and chemokines, and that tumor-promoting inflammation enables cancer risk and subsequent development [4-6]. For instance, the family of TNF- $\alpha$ receptor-associated factor (TRAF) was found to be associated with lung cancer development, and, more specifically, the suppression of TRAF6 promoted the apoptosis of lung cancer cells [7]. Moreover, TRAF2 was reported to be involved in the signal transduction associated with lung cancer development through its interaction with the TRAF-interacting protein with a forkhead-associated (FHA) domain (TIFA) [8-10].

TIFA interacts with TRAF6 and modulates the activation of the IkB kinase (IKK) complex, which subsequently activates I $\kappa \mathrm{B}$ through phosphorylation and ubiquitination-dependent

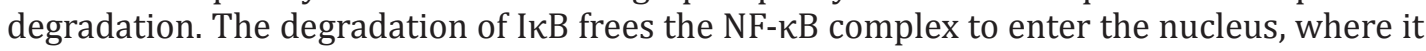
initiates the transcription of downstream factors that regulate many cell functions, including its inflammatory and immunological response, as well as cell survival and proliferation [11]. Consistently, NF- $\kappa$ B (p65) phosphorylation was detected in different types of cancer cells, and established to upregulate genes that promote cell proliferation or protect cells from apoptosis $[12,13]$.

The role of TIFA in cancer development has been previously reported in several types of cancer, including hepatocellular cancer and acute myeloid leukemia $[14,15]$. However, the results of these examinations were controversial. Therefore, the aim of the present study was to investigate the expression of TIFA in lung adenocarcinoma tissues and elucidate the role of TIFA in the modulation of lung cancer cell proliferation, migration, and survival, as well as its potential mechanisms of action.

\section{Materials and Methods}

\section{Materials}

A rabbit anti-human TIFA monoclonal antibody and an anti-GAPDH antibody were purchased from Proteintech (Wuhan, Hubei, China). A SABC immunohistochemistry kit and DAB kit were obtained from Beijing Zhongshan-Golden Bridge (Beijing, China).

An immortalized bronchial epithelial cell line (BEAS2B), an alveolar carcinoma cell line (A549), and lung cancer cell lines (H1299, H1975, and HCC827) were purchased from American Type Culture Collection (ATCC, Manassas, VA, USA). Fetal calf serum (FCS), DMEM, and Opti-MEM were purchased from ThermoFisher Scientific, GIBCO (USA).

TIFA-siRNA and transfection reagent (Lenti-Easy Packaging mix) were bought from Shanghai Jikai Biotech (Shanghai, China), and TRIzol (Invitrogen) and One Step SYBR PrimeScript RT-PCR kit II from Takara (Japan). Annexin V-FITC Apoptosis Detection kit was acquired from Abcam (USA).

\section{Cell culture}

All cell lines were cultured on DMEM supplemented with $10 \%$ fetal calf serum, penicillin/streptomycin, and fungizon. Every 2-3 days, the medium was replaced with fresh medium, and the cells were passaged at confluence.

\section{Immunoblotting}

Cells were harvested using cell lysis buffer, followed by centrifugation of the cell lysates at 12, $000 \mathrm{rpm}$ $\mathrm{x} 20 \mathrm{~min}$ at $4{ }^{\circ} \mathrm{C}$. Then, the protein concentration in the supernatants was determined by using a BCA kit (Pierce Biotechnology, Rockford, IL, USA). Protein (50 $\mu$ g in $5 \mu \mathrm{L}$ ) was further loaded into 10\% SDS-PAGE gel, and electrophoresis was performed. Next, the samples were transferred onto PVDF membranes, which were blocked with 5\% milk in a phosphate-buffered saline-tween (PBS-T) solution for $1 \mathrm{~h}$ at room temperature. 


\section{Cellular Physiology Cell Physiol Biochem 2018;47:2097-2108

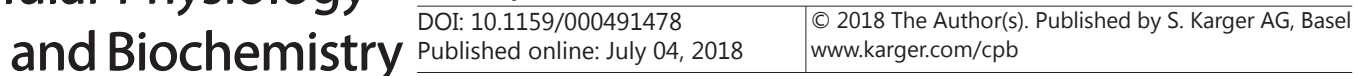 \\ Men et al.: TIFA in Lung Adenocarcinoma}

The primary antibody used at the concentration recommended by the manufacturer was allowed to react with target proteins overnight at $4{ }^{\circ} \mathrm{C}$. After washing with PBS-T, the membrane was incubated with HRPconjugated $2^{\text {nd }}$ antibody at room temperature for $1.5 \mathrm{~h}$. Further, after washing, the targeted protein bands were visualized using ECL detection. GAPDH was utilized as a loading control, and the intensity of each band was quantified using image analysis software (ImageJ, National Institutes of Health, Bethesda, MD, USA).

\section{Immunocytochemistry}

Cells were cultured on cover slips and fixed with $4 \%$ methanol at $4{ }^{\circ} \mathrm{C}$ for $20 \mathrm{~min}$. After washing, the cells were permeabilized with $0.2 \%$ Triton X-100 for $10 \mathrm{~min}$ at room temperature. Then, blocking was performed with goat serum, and anti-p65-antibody (Abcam, Cat \#: ab16502) was applied overnight at $4{ }^{\circ} \mathrm{C}$. The second antibody was added on the next day, followed by visualization of the protein expression using DAB staining and nuclear counterstaining with hematoxylin.

\section{Real-time RT-PCR}

The expression of TIFA mRNA was quantified by real-time RT-PCR. Briefly, total RNA was extracted from the cell lines using TRIzol reagent. After quantification, a total of 10 ng RNA was used for cDNA synthesis, followed by real-time PCR using One Step SYBR® PrimeScript ${ }^{\text {TM }}$ RT-PCR Kit II according to the manufacturer's instructions. GPDH was employed as an internal control. The sequences of the primers used were as follows: TIFA-forward: 5'-ATGACCAGTTTTGAAGATGCTG-3'; TIFA-backward: 5'-TCCCAAAGCAGTTCTCCGACAG-3'; GAPDH-forward: 5'-TGTGGGCATCAATGGATTTGG-3'; GAPDH-backward: 5'-ACACCATGTATTCCGGGTCAAT-3'. Relative quantification of TIFA mRNA was conducted using the $2^{-\triangle \Delta C T}$ method, followed by normalization using the internal control.

RNAi suppressed TIFA expression

H1975 and HCC827 cells were plated at a density of $2 \times 10^{5}$ per a 60 -mm dish. After 24-h culture, the cell culture medium was changed to Opti-MEM, and the cells were incubated for $2 \mathrm{~h}$. The cells were then transfected with TIFA-siRNA or control-siRNA $(50 \mu \mathrm{L})$ in $1.5 \mathrm{~mL}$ of Opti-MEM medium and $60 \mu \mathrm{L}$ of Lipofectamine 2000 in $1.5 \mathrm{~mL}$ of Opti-MEM (total $3 \mathrm{~mL}$ of Opti-MEM) for $4 \mathrm{~h}$. After brief washing with PBS, the cells were cultured for additional $48 \mathrm{~h}$ in 10\%FCS-DMEM medium.

\section{Cell proliferation assay}

Cell proliferation was assessed using the CCK8 method following the manufacturer's instructions (Beyotime Biotechnology). Briefly, the cells were plated into a 96-well tissue culture plate at a density of $10^{5}$ cells/well in $100 \mu \mathrm{L}$ of cell culture medium. Further, cell proliferation was assessed on day $1,2,3,4$, and 5 by adding 10\% CCK 8 and incubation for $4 \mathrm{~h}$. The optical density was measured at a wavelength of $450 \mathrm{~nm}$. Medium plus CCK8 without cells was used as a blank sample, and the rate of cell proliferation was calculated.

Flow cytometry analysis for an assessment of cell-cycle arrest and apoptosis

Cell-cycle arrest and apoptosis were assessed by flow cytometry. Briefly, after transfection with TIFA-

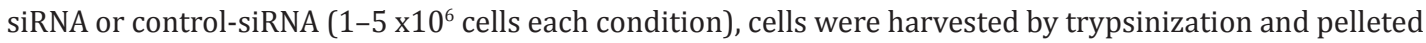
by centrifugation at $1000 \mathrm{rpm} \times 5 \mathrm{~min}$. The cells were then washed once with $3 \mathrm{~mL}$ of PBS, followed by fixation with $70 \%$ ethanol at $4{ }^{\circ} \mathrm{C}$ for $2 \mathrm{~h}$. After washing with PBS, the cells were stained with $1 \mu \mathrm{g} / \mathrm{mL}$ of propidium iodide (PI) in $1 \mathrm{~mL}$ of PBS at $4{ }^{\circ} \mathrm{C}$ for $30 \mathrm{~min}$. Further, their cell cycle was examined by flow cytometry.

Apoptosis was evaluated using an Annexin-V kit following the manufacturer's instructions. Briefly, after trypsinization, the cells were pelleted and washed once with cold PBS, followed by centrifugation at $4{ }^{\circ} \mathrm{C}$ (2000 rpm x $\left.5 \mathrm{~min}\right)$. The cells (1-5 x $10^{6}$ cells each condition) were then re-suspended with 300 $\mu \mathrm{L}$ of binding buffer, followed by the addition of $5 \mu \mathrm{L}$ of Annexin-V-FITC solution and incubation at room temperature in the dark for $15 \mathrm{~min}$. The nuclei were stained with $5 \mu \mathrm{L}$ of PI for 5 min. Finally, another 200 $\mu \mathrm{L}$ of binding buffer was added, and apoptosis was evaluated by flow cytometry.

Trans-well migration and wound-healing assay

For the trans-well assay, each well was coated with $30 \mu \mathrm{L}$ of Matrigel for $2 \mathrm{~h}$ at $37^{\circ} \mathrm{C}$. Cells were then added into the top wells at a density of $5 \times 10^{5}$ cells $/ \mathrm{mL}, 100 \mu \mathrm{L} /$ well. In the bottom well, medium containing 


\section{Cellular Physiology Cell Physiol Biochem 2018;47:2097-2108 \begin{tabular}{l|l} 
DOI: 10.1159/000491478 & and Biochemistry \\
Published 2018 The Author(s). Published by S. Karger AG, Basel \\
www.karger.com/cpb
\end{tabular} \\ Men et al.: TIFA in Lung Adenocarcinoma}

$20 \%$ FCS was added as a chemoattractant ( $500 \mu \mathrm{L} /$ well). Further, the cells were incubated and allowed to migrate for $24 \mathrm{~h}$. On the next day, the Matrigel and non-migrated cells in the top wells were wiped off, and the migrated cells were air-dried and transferred onto a 24 -well plate. Crystal violet (1\%) was then added into the 24 -well plate $(500 \mu \mathrm{L} /$ well $)$, followed by incubation at $37^{\circ} \mathrm{C}$ for $30 \mathrm{~min}$. After washing with PBS, the number of migrated cell was determined by counting under a high-power field ( $200 \mathrm{x})$; the procedure was performed in three different fields per well.

To conduct the wound-healing assay, cells were plated into a 6-well tissue culture plate and allowed to reach $60 \%$ confluence. Cell layer was wounded with a pipette tip, and the floating cells were removed by washing once with PBS. The cells were then cultured in normal medium for $24 \mathrm{~h}$. After fixation, the woundhealing was assessed by measuring the distance between the lines of the wounded area under a microscope at $100 \times$ magnification.

\section{Statistical analysis}

Statistical analysis was performed using SPSS23.0 software. Data were expressed as mean \pm standard deviation (SD). Student's $t$-test or one-way ANOVA were employed to examine the difference among groups. A $p$ - value $<0.05$ was considered significant.

\section{Results}

\section{TIFA expression in the lung cancer specimens}

A total of $63(54.3 \%)$ lung cancer specimens out of the 116 specimens from lung cancer tissues and their adjacent normal lung tissues, were strongly positive (Fig 1A and 1B) or weakly positive to TIFA (Fig. 1C and 1D), whereas most of the samples from the adjacent lung tissues were TIFA-negative (Fig. 1E and 1F), and less than 35 of them (30.1\%) were positive. A significant difference was found in TIFA positivity between the two groups $(p<0.05)$. As can be observed in Table 1, the patients with positive TIFA expression were with a later stage (III-IV) and lower differentiation of cancer as well as a higher death rate $(p<0.05)$.

The results of the univariate regression analysis of the relationship of TIFA expression and patient's general information, and the pathological characteristics of lung adenocarcinoma (Table 2) indicated that the expression level of TIFA in the lung cancer tissues was associated

Fig. 1. Expression of TIFA in lung cancer tissues. The expression of TIFA was evaluated in cancer tissues and their adjacent normal tissues obtained from lung cancer patients. The data presented are representative of the 116 specimens. Panels A and B: strong TIFA-positive immunostaining in one of the representative lung cancer specimens at $200 \mathrm{x}$ (A) and 400x (B) magnification; Panels C and D: weak TIFA-positive immunostaining in one of the representative lung cancer specimens at 200x (C) of and 400x (D) magnification; Panels $E$ and F: TIFAnegative immunostaining in the adjacent normal lung tissue of the lung cancer tissue depicted in Panels A and $\mathrm{B}$, at $200 \mathrm{x}$ of $(\mathrm{E})$ and $400 \mathrm{x}(\mathrm{F})$ magnification.

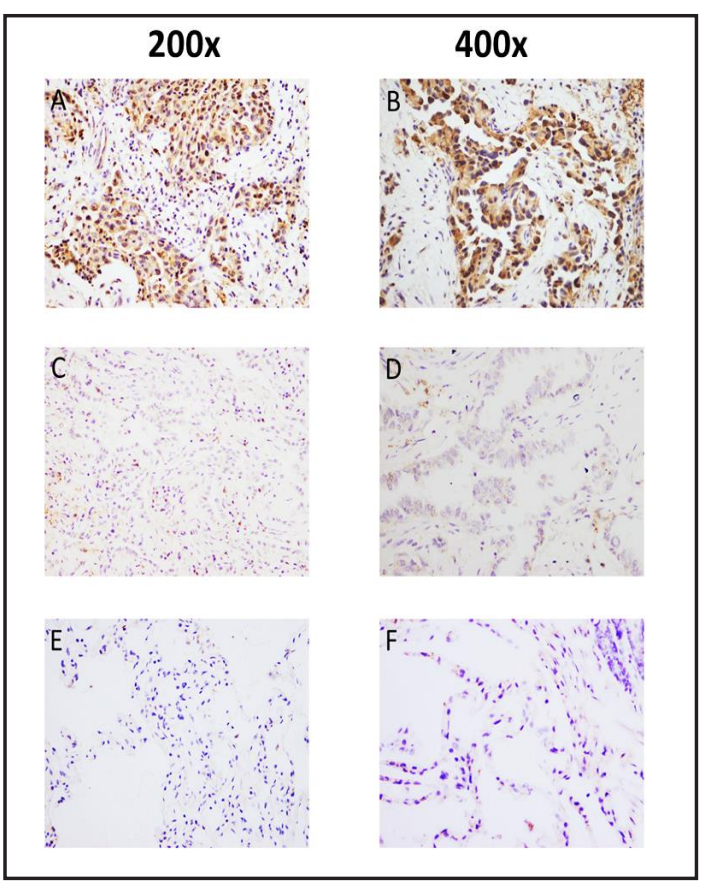


Fig. 2. TIFA expression and overall survival of lung cancer patients. Comparison between the overall survival rates of patients with TIFA-positive and TIFA-negative expression, evaluated by the KaplanMeier survival curve.

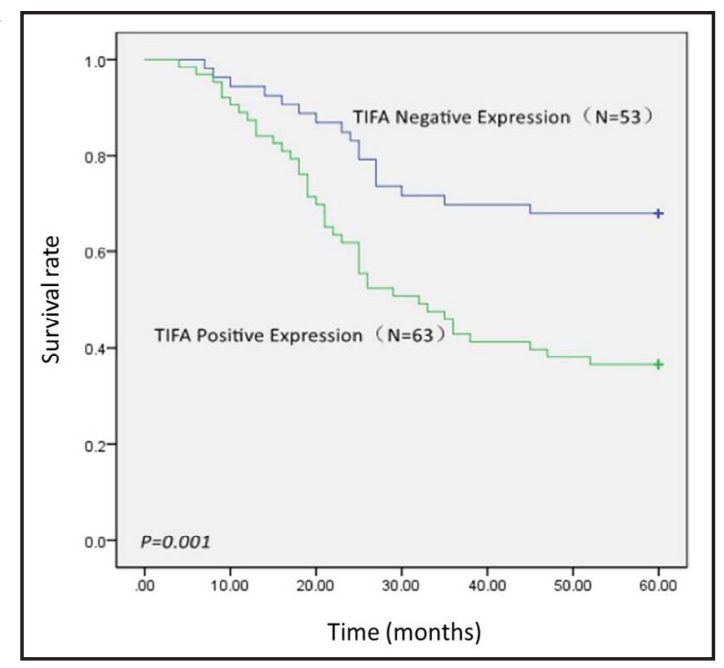

with the TNM stage ( $p=0.023$ ), cancer cell differentiation ( $p=0.007)$, and survival ( $p$ $=0.001$ ). In contrast, it was not associated with sex, age, and cigarette smoking history or tumor size. The multivariate regression analysis suggested that TIFA positivity and TNM stage significantly affected the patient's survival ( $p=0.028$ and 0.014 , respectively). Furthermore, the Kaplan-Meier survival curve revealed that the survival rate of the TIFA-positive patients was significantly lower than that of the TIFA-negative patients (Fig. 2, $p<$ $0.05)$.

\section{Role of TIFA in cancer cell proliferation and survival}

To investigate the role of TIFA in the regulation of cancer cell proliferation and metastasis, four different lung cancer cell lines (A549, H1299, H1975, and HCC827) were used for in vitro study and compared with a line of immortalized normal bronchial epithelial cells (BEAS2B). The expression levels of TIFA protein (Fig. $3 \mathrm{~A}$ and $\mathrm{BB}$ ) in the adenocarcinoma cell lines were significantly higher than they were in the normal cell line $(0.84 \pm 0.19$ in HCC827, $0.91 \pm 0.17$ in H1975, and $0.60 \pm 0.13$ in $\mathrm{H} 1299$ vs $0.38 \pm 0.08$ in BEAS2B, $p<0.05$ or 0.01 ). Consistently, the expression of TIFA mRNA (Fig. 3C) was also significantly higher in the adenocarcinoma cells lines than in the normal cell line $(1.33 \pm 0.05$ in HCC827, $1.36 \pm 0.09$ in H1975, $1.21 \pm 0.06$ in H1299, and 1.08 \pm 0.05 in A549 vs $1.00 \pm 0.07$ in BEAS2B, $p<0.05$ or 0.01 ).

Additionally, using RNAi, we found that the expression of TIFA was suppressed in the H1975 and HCC827 cancer cell lines (Fig. 4). The suppression of TIFA by siRNA in the two above-mentioned cell lines resulted in significant inhibition of cell proliferation from day 2 to day 5 (Fig. 5A and 5B, respectively, $p<0.05$ ). Furthermore, this inhibitory effect exerted by siRNA lines led to the apoptosis of the cancer cell, evidenced by the increased Annexin- 


\section{Cellular Physiology Cell Physiol Biochem 2018;47:2097-2108 \begin{tabular}{l|l} 
DOI: 10.1159/000491478 & O 2018 The Author(s). Published by S. Karger AG, Basel \\
www.karger.com/cpb
\end{tabular}

Fig. 3. Comparison of TIFA in lung cancer cell lines and normal bronchial epithelial cells. Panels $A$ and B: protein levels of TIFA in four cancer cell lines (A549, H1299, H1975, and HCC827) and one immortalized normal bronchial epithelial cell line (BEAS-2B). Total protein from cell lysate was harvested and immunoblotted for TIFA as described in the methods. Representative image data (A) and semi-quantified data of three separate immunoblotting (B) were presented. Panel C: expression of TIFA mRNA in the four cancer cell lines and BEAS-2B cells. Total RNA was extracted, and real-time RT-PCR was performed as described

in the methods. Data are presented as an average of three separate experiments. ${ }^{*} \mathrm{p}<0.05$; ${ }^{* *} \mathrm{p}<0.01$ compared to BEAS-2B cells.

Fig. 4. Suppression of TIFA by siRNA. GFPtagged TIFA-specific and control siRNAs were transfected into the lung cancer cell lines (H1975 and HCC827) as described in the methods. Panel A: representative image of the cells under a regular and fluorescence microscope after the transfection with TIFA-specific siRNA; Panel B: Representative data of the immunoblotting of TIFA in the cells transfected with the control siRNA or TIFA-specific siRNA.

Fig. 5. Suppression of TIFA by siRNA and its effect on cell proliferation. Specific siRNA targeting TIFA and non-specific control siRNA were transfected into the lung cancer cell lines H1975 (Panel A) and HCC827 (Panel B), and cell proliferation was then assessed as described in the methods. Vertical axis: optical density at a wavelength of $490 \mathrm{~nm}$ corresponding to the cell number; horizontal axis:
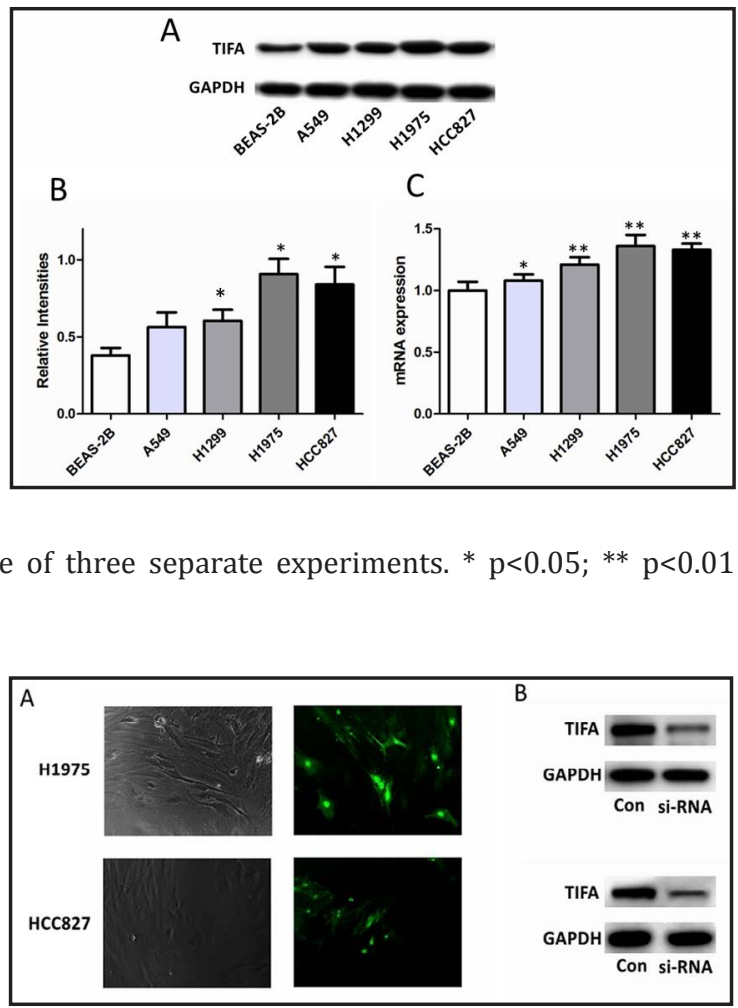

time (day). Data are presented as an average of three separate experiments. ${ }^{*} \mathrm{p}<0.05 ;{ }^{* *} \mathrm{p}<0.01$ between the two groups at each time point.

V-positive in the cells lacking TIFA (H1975: $33.4 \pm 3.8 \%$; HCC827: $34.8 \pm 2.6 \%$ ), whereas no such effect was observed in the control cells (H1975: $8.8 \pm 5.3 \%$, Fig. 6A and 6B, $p<0.05$; HCC827: $10.6 \pm 1.1 \%$, Fig $6 \mathrm{C}$ and $6 D, p<0.05)$. Cell cycle examination by flow cytometry also confirmed that the percentages of G0/G1 stage cells were increased in $\mathrm{H} 1975(78.3 \pm 1.8 \%)$ and HCC827 (79.6 $\pm 3.8 \%)$ after the transfection with TIFA-siRNA and were significantly higher than those in the cells transfected with control-siRNA (56.3 $\pm 3.7 \%$ and $60.3 \pm 3.7 \%$, respectively, $p<0.05$, Fig. 7).

\section{Role of TIFA in the modulation of cancer cell migration}

The suppression of TIFA by siRNA in the H1975 or HCC827 cell lines resulted also in a significant reduction in cell migration (H1975: $14.0 \pm 3.5$ cells/HPF vs $35.0 \pm 2.5$ cells/ HPF, $p<0.05$; HCC827: $15.6 \pm 2.1$ cells/HPF vs $28.3 \pm 2.5$ cells/HPF, $p<0.01$, Fig. 8A and $8 \mathrm{~B})$. Furthermore, the wound-healing assay revealed that the cells lacking TIFA had a more significantly reduced ability of wound-healing (H1975: 228.0 $\pm 2.5 \mu$ m; HCC827: $296.7 \pm 4.5$ $\mu \mathrm{m})$ than the control lung cancer cells, in which TIFA was overexpressed (H1975: $131.3 \pm 7.6$ $\mu \mathrm{m}$, Fig 9A and 9B; HCC827: $190.0 \pm 2.9 \mu \mathrm{m}$, Fig 9C and 9D, $p<0.01$, respectively). 
Fig. 6. Suppression of TIFA by siRNA and its effect on cell survival. Specific siRNA targeting TIFA and non-specific control siRNA were transfected into the lung cancer cell lines H1975 (Panels A, B \& C) and HCC827 (Panels D, E \& F), and apoptosis was then assessed by the quantification of Annexin-Vpositive cells using flow cytometry. Panels A and B: representative image data of the flow cytometry analysis of the H1975 cells transfected with control siRNA (A)

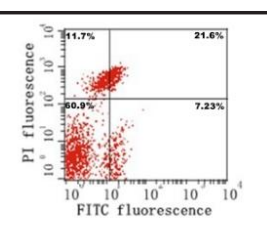

A. Control siRNA (H1975)

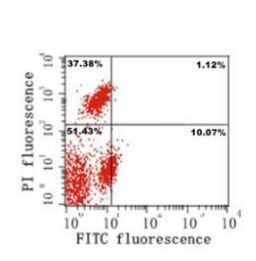

D. Control siRNA (HCC827)

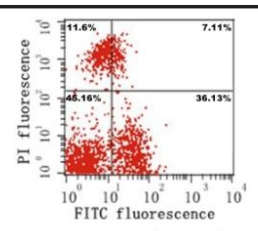

B. TIFA-SiRNA (H1975)

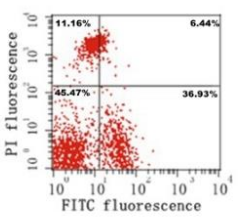

E. TIFA-siRNA (HCC827) F. Average of 3 separate experiments and TIFA-siRNA (B); Panels D and E: representative image data of the flow cytometry analysis of the HCC827 cells transfected with control siRNA (D) and TIFA-siRNA (E); Panels C and F: quantitative representation of three separate experiments. * $\mathrm{p}<0.05$; ** $\mathrm{p}<0.01$ compared to the cells of the control.

Fig. 7. Suppression of TIFA by siRNA and its effect on cell-cycle arrest. Specific siRNA targeting TIFA and non-specific control siRNA were transfected into the lung cancer cell lines H1975 (Panels A, B \& C) and HCC827 (Panels D, E \& F).Then, the cell cycle was assessed by flow. Panels $A$ and B: representative image data of the flow cytometry analysis of H1975 cells transfected with control siRNA (A) and TIFA-siRNA (B); Panels D and E: representative
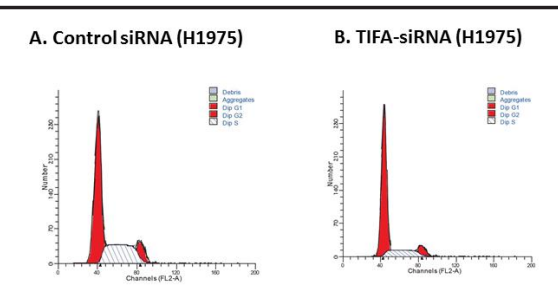

C. Average of 3 separate experiments
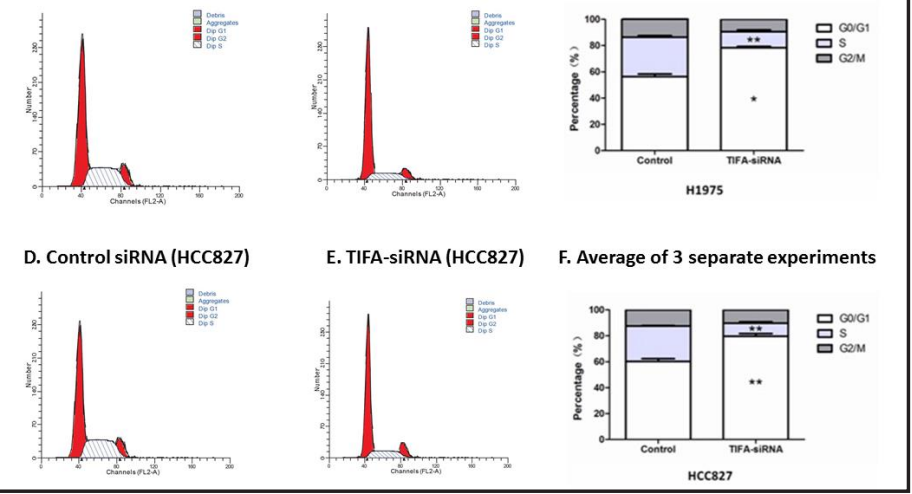
image data of the flow cytometry analysis of HCC827 cells transfected with control siRNA (D) and TIFA-siRNA (E); Panels C and F: quantitative presentation of three separate experiments. ${ }^{*} \mathrm{p}<0.05 ;{ }^{* *} \mathrm{p}<0.01$ compared to the cells of the control.

Fig. 8. Suppression of TIFA by siRNA and its effect on cell migration. After the transfection of TIFA-specific siRNA and control siRNA, the ability of cell migration was assessed by trans-well assay as described in the methods. Panel A: representative image data of cells migrated through the trans-well

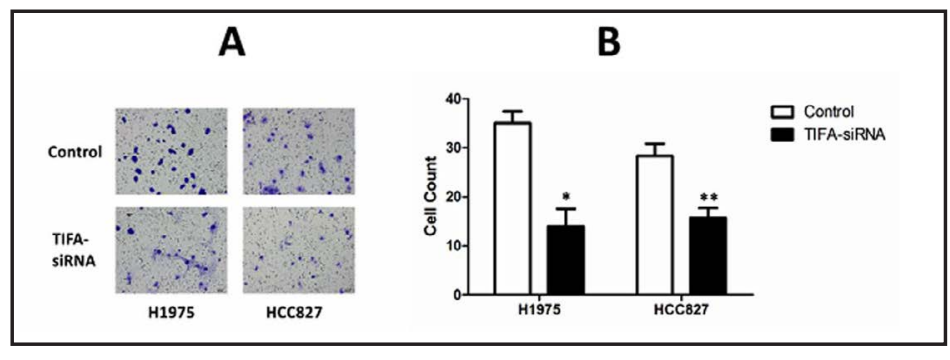
membrane; Panel B: quantitative representation of the cell number obtained from the counting in three separate experiments. ${ }^{*} \mathrm{p}<0.05$; ${ }^{* *}$ $\mathrm{p}<0.01$ compared to the lung cancer cell lines transfected with the control siRNA. 


\section{Effect of TIFA on proteins regulating cell cycle and survival}

As illustrated in Fig. 10, the proteins associated with cell cycle (p21, cyclin D1, and CDK4), cell survival (p53, Bcl-2, and caspase-3), and cell migration (HMGB1) were expressed and easily detectable by immunoblotting in the two studied cancer cell lines (H1975 and HCC827). The suppression of TIFA by siRNA resulted in significant upregulation of p53 and increased levels of cleaved caspase-3 ( $p<0.05$, Fig. 10). In contrast, this inhibitory effect caused considerable downregulation of Bcl-2 protein $(p<0.05$, Fig. 10). Similarly, the inhibition of the expression of TIFA by siRNA led to a substantial reduction in the levels of cyclin D1 and CDK4 ( $p<0.05$, Fig. 10), but contributed to a significant increase in p21 levels $(p<0.05$, Fig. 10). In addition, HMGB1 protein was significantly more inhibited in the H1975 (Fig. 10A and C) and HCC827 (Fig. 10B and D) cells lacking TIFA than in the control cells ( $p$ $<0.05$, Fig. 10).

Next, we used immunoblotting to investigate the role of TIFA on the regulation of the

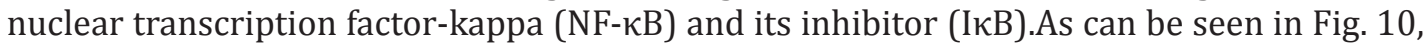
positive staining of p-IKKß and p-p65 was detected in the two cancer cell lines (H1975: Fig. 11A and C; HCC827: Fig. 11B and D) as evidenced by the phosphorylation of IKKß (p-IKKß), $\mathrm{I} \kappa \mathrm{B} \alpha(\mathrm{p}-\mathrm{I} \kappa \mathrm{B} \alpha)$, and p-65 (p-p65). However, the levels of p-IKKß, p-I $\mathrm{B} \alpha$, and p-p65 were significantly decreased in the cells transfected with TIFA-siRNA $(p<0.05$, Fig. 11).

Fig. 9. Suppression of TIFA by siRNA and its effect on wound-healing. After the transfection of TIFAspecific siRNA and control siRNA, the ability of cell migration was further assessed by the "woundhealing" assay in the two cell lines of lung cancer, H1975 (Panels A and C) and HCC827 (Panels B and D), as described in the methods; Panels A and $\mathrm{C}$ : representative image data of cells migrated during the wound-healing; Panels B and D: quantitative representation of the wound distance measurement from three separate experiments. ** $\mathrm{p}<0.01$ compared to the lung cancer cell lines transfected with the control siRNA.

Fig. 10. Role of TIFA in the regulation of proteins associated with cell survival and cell-cycle arrest. Specific siRNA targeting TIFA and non-specific control siRNA were transfected into the lung cancer cell lines H1975 (Panels A \& C) and HCC827 (Panels B \& D). Total protein was extracted and subjected to immunoblotting of p53, Bcl-2, caspase-3, p21, cyclin D1, CDK4, and HMGB1. GAPDH was used as a loading control. Panels A and B: representative image data of immunoblotting; Panels C and D: quantitative representation of three separate experiments. ${ }^{*} \mathrm{p}<0.05 ;{ }^{* *} \mathrm{p}<0.01$ compared to the cells of the control.
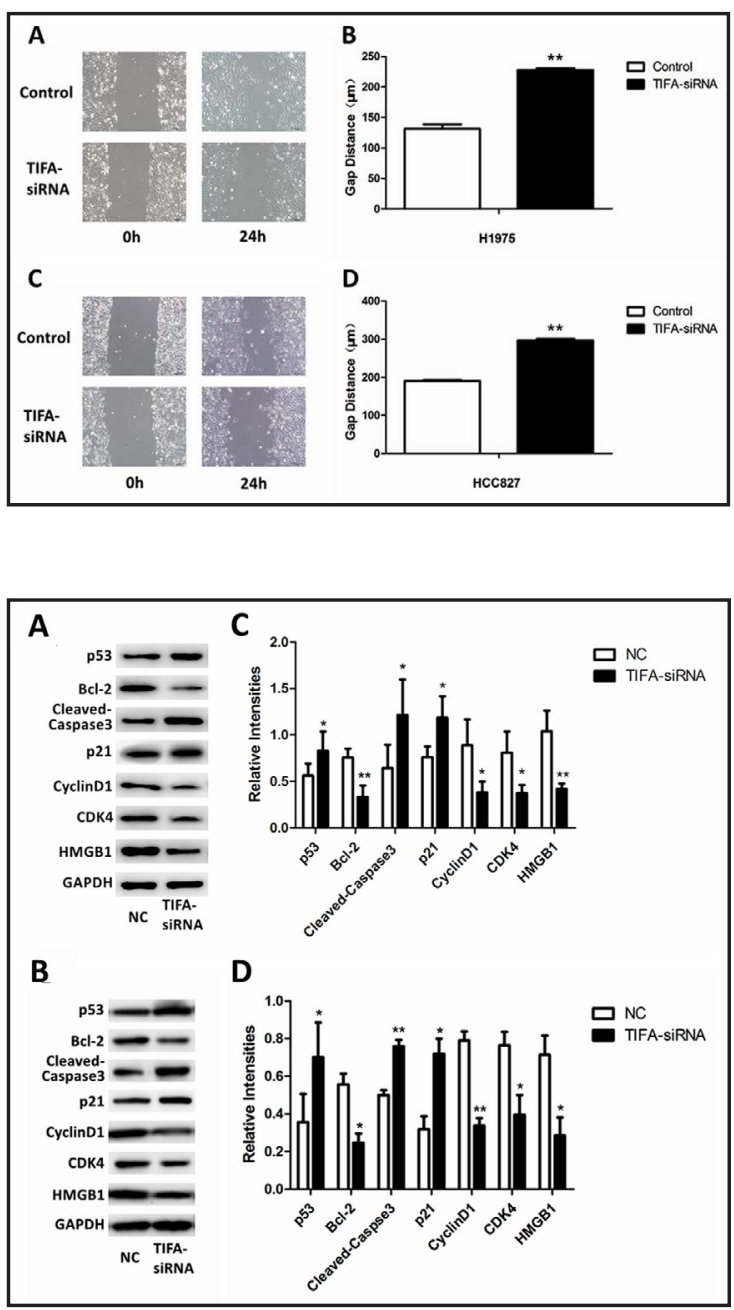
Fig. 11. Role of TIFA in the regulation of NF$\kappa \mathrm{B}$ signaling. Specific siRNA targeting TIFA and non-specific control siRNA were transfected into the lung cancer cell lines, H1975 (Panels A \& C) and HCC827 (Panels B \& D). Total protein was extracted and subjected to immunoblotting of total and phosphorylated IKKß, I $\mathrm{B}$, and p65. GAPDH was used as a loading control. Panels A and B: representative image data of immunoblotting; Panels C and D: quantitative representation of three separate experiments. ${ }^{*} \mathrm{p}<0.05 ;{ }^{* *} \mathrm{p}<0.01$ compared to the cells of the control.

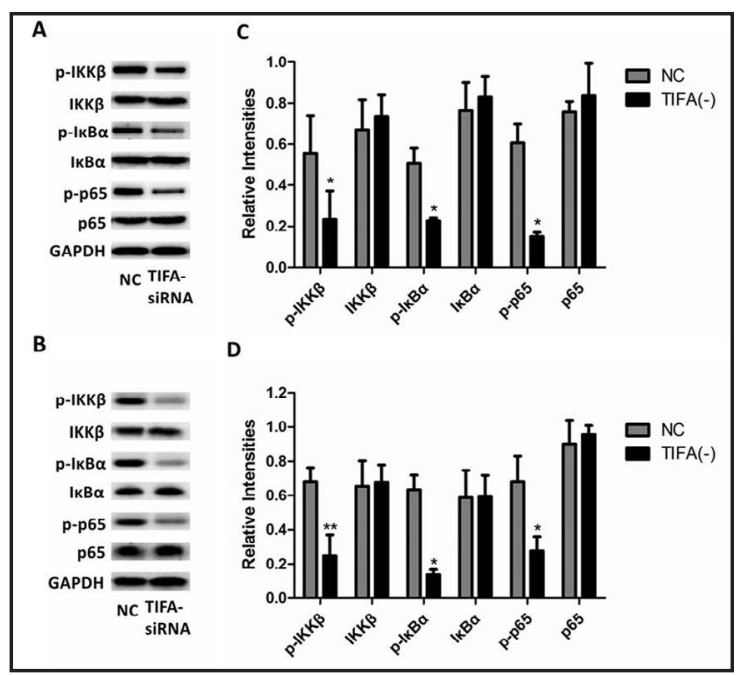

\section{Discussion}

In the present study, we investigated the expression of TIFA in lung cancer tissues and its role in the mediation of cancer cell survival and invasion. Our findings showed that the expression of TIFA in the lung cancer tissues was significantly higher than that in the adjacent healthy tissues. Moreover, the overall survival rate was considerably lower in the lung cancer patients with positive expression of TIFA than in the patients with negative TIFA expression. The suppression of TIFA by siRNA in the lung cancer cell lines resulted in significant inhibition of cell proliferation and migration but significantly increased apoptosis. In addition, the inhibition of TIFA in the lung cancer cell lines caused an increase in the levels of p53, p21, and cleaved-caspase-3, but decreased those of Bcl-2, cyclin D1, CDK4, and HMGB1. The phosphorylation of IKKß, IKB $\alpha$, and p- 65 was significantly suppressed in the lung cancer cell lines (H1975 and HCC827) after TIFA silencing by siRNA. These findings indicate that TIFA is associated with the overall survival rate of lung cancer patients and might increase lung cancer cell survival and invasion via inhibiting the apoptosis of lung cancer cells.

Lung cancer is the leading cause of cancer-associated death and the second most common newly diagnosed cancer in the world $[1,16]$. In China, the mortality of lung cancer has been increasing over the last decades [17], which is partially due to the lack of a specific biomarker or effective detection methods for its early diagnosis. Thus, biomarkers and targeted therapy of lung cancer has been extensively studied in the recent years. In the current study, we investigated the expression of TIFA in lung cancer tissues and its potential role in the prediction of patient outcomes. We found that the expression of TIFA was significantly increased in the lung adenocarcinoma tissues, and the TIFA-positive patients had a survival rate that was significantly lower than that of the lung cancer patients with negative TIFA expression. Based on these findings, we can speculate that the higher TIFA protein level in the lung cancer tissue could also serve as a novel biomarker that predicts the clinical outcome of patients with lung adenocarcinoma.

TIFA is a TRAF6-interacting protein, which was identified and first reported by Takatsuna et al. in 2003 [10]. Specifically, TIFA activates NF- $\kappa B$ and JNK by linking TRAF 6 to IRAK-1 in the IL-1 pathway [10]. TRAFs are a family of proteins that participate in the signal transduction of TNF- $\alpha$ and IL-1 receptor/Toll-like receptor (IL-1R/TLR) super-families, and high expression of TRAF6 has been reported in lung cancer patients [18-21]. TIFA seems to be involved in the regulation of the general response to pro-inflammatory stress though LPD-mediated signaling [22]. However, controversial results have been obtained in studies on the association of TIFA and cancer cell survival and proliferation. For example, Wei et al. 
reported that TIFA was expressed in acute myeloid leukemia (AML), and suppression of TIFA in AML cell lines by siRNA successfully decreased the leukemic cell growth and chemotherapy resistance [15]. By contrast, Shen et al. reported that the expression of TIFA in hepatocellular carcinoma (HCC) cells was lower than that in normal liver control cells; furthermore, the reconstitution of TIFA expression promoted the apoptosis of the HCC cells [14].

Conversely, here we report that the expression of TIFA in four lung cancer cell lines (A549, H1299, H1975, and HCC827) was higher than that in immortalized normal bronchial epithelial cells (BEAS2B). Furthermore, the suppression of TIFA by siRNA in the lung cancer lines resulted in significant inhibition of cell proliferation and ability of cell migration. These findings suggest that the role of TIFA vary in different tissue types of cancer cells; that is, while it exerts tumor-suppressive effects in hepatocellular carcinoma, oncogenic influence is established in lung cancer and hematopoietic malignancies.

While the precise causes and mechanisms of the aforementioned difference remain to be further investigated, the regulation of apoptosis by TIFA may play a key role in the survival of cancer cells. In this content, TIFA interacts with TRAF6 and modulates the oligomerization and ubiquitination of TRAF 6 to activate the IKB kinase (IKK) complex, followed by subsequent phosphorylation and ubiquitination-dependent degradation of the inhibitor of $\kappa B$ (I $\kappa B$ ), resulting in nuclear translocation of NF- $\mathrm{KB}$, which activates downstream factors [11].

$\mathrm{NF}-\kappa \mathrm{B}$ controls various aspects of the biological responses in a variety of cells, including immune responses, as well as cell survival, proliferation, and differentiation [23]. An increasing number of recent evidence indicates that NF- $\kappa \mathrm{B}$ is aberrantly activated and cross-talks with other signaling molecules and pathways in chronic inflammation and a spectrum of malignancies [24]. Consistently, the phosphorylation of p65 in cancer cells was shown to protect tumor cells from apoptosis and increase their resistance to chemotherapy and ionizing radiation through regulation of the balance between pro-apoptotic and antiapoptotic factors $[25,26]$. In line with this concept, the role of NF- $\kappa B$ and its downstream molecules in the modulation of lung cancer cell cycle and survival was explored in this study. In agreement with previous reports $[15,27]$, we established that the silencing of TIFA promoted apoptosis and inhibited the cell migration of the lung cancer cell lines. Moreover, it attenuated the phosphorylation of IKK, IкB, and p65, and decreased the levels of Bcl-2, cyclin D1, and CDK4, but increased those of p21, p53, and cleaved caspase-3. These findings suggest that TIFA promotes lung cancer cell survival presumably through constitutive phosphorylation of the proteins involved in the NF- $\mathrm{KB}$ signaling pathway; it subsequently stimulates the pro-survival responses and activates anti-apoptotic signals.

Nevertheless, the current study has some limitations. First, the number of lung cancer patients was small. Second, this investigation was a retrospective study. Thus, prospective studies with larger numbers of cases and double-blinded control studies are required to further confirm the current findings. Third, Annexin-V assay was used in the present study to assess apoptosis, but Annexin- $V$ detection can be used only to evaluate the early events in the process of apoptosis (the abnormal permeability and injury of the cell membrane). However, it does not detect the late-stage events of apoptosis, including DNA damage and break, which could only be detected by TUNEL and COMET assays. Finally, the "normal" lung tissues used for the comparative analysis of TIFA expression by immunohistochemistry were from the adjacent "normal" lung tissues of the same lung cancer patients, which might not have truly represented the normal lung tissue.

Taken together, the results of the present study reveal that the expression of TIFA in lung cancer tissues is higher than that in the adjacent normal lung tissues. In addition, TIFA was constitutively expressed in the lung cancer cell lines not only at the protein level, but also at the transcriptional level, with statistically significant higher values than those in the normal bronchial epithelial cell line. It is noteworthy that the patients with positive TIFA expression had significantly lower overall survival rates. The suppression of TIFA in the lung cancer cell lines by siRNA resulted in significant attenuation of cell proliferation and migration, as well as promotion of cancer cell apoptosis and cell-cycle arrest. Additionally,

\section{KARGER}


NF- $\kappa$ B (p65) phosphorylation was detected in the lung cancer cell lines, and the suppression of TIFA caused attenuation of $\mathrm{p} 65$ phosphorylation. The inhibition of TIFA by siRNA also led to alteration of apoptosis- and cell cycle-associated protein levels, including p53, caspase-3, $\mathrm{Bcl}-2$, p21, cyclin D1, and CDK4. These findings suggest that TIFA may act as an anti-apoptotic factor in lung cancer cells and can thus serve as a biomarker for the prediction of lung cancer survival.

\section{Acknowledgements}

This study was supported by the Natural Science Foundation of Liaoning Province (Grant no. 2015020561) and the Fund for Scientific Research of The First Hospital of China Medical University (Grant no. fsfh1514).

\section{Disclosure Statement}

The authors declare to have no competing interests.

\section{References}

1 Siegel RL, Miller KD, Jemal A: Cancer statistics, 2017 CA Cancer J Clin 2017;67:7-30.

2 Ettinger DS, Wood DE, Akerley W, Bazhenova LA, Borghaei H, Camidge DR, Cheney RT, Chirieac LR, D’Amico TA, Demmy TL, Dilling TJ, Dobelbower MC, Govindan R, Grannis FW, Jr., Horn L, Jahan TM, Komaki R, Krug LM, Lackner RP, Lanuti M, Lilenbaum R, Lin J, Loo BW, Jr., Martins R, Otterson GA, Patel JD, Pisters KM, Reckamp K, Riely GJ, Rohren E, Schild SE, Shapiro TA, Swanson SJ, Tauer K, Yang SC, Gregory K, Hughes M, National comprehensive cancer n: Non-small cell lung cancer, version 6.2015. J Natl Compr Canc Netw 2015;13:515-524.

-3 Roque Ramos L, DiMaio CJ, Sachar DB, Atreja A, Colombel JF, Torres J: Autoimmune pancreatitis and inflammatory bowel disease: Case series and review of the literature. Dig Liver Dis 2016;48:893-898.

4 Crusz SM, Balkwill FR: Inflammation and cancer: Advances and new agents. Nat Rev Clin Oncol 2015;12:584-596.

5 Liao C, Yu Z, Guo W, Liu Q, Wu Y, Li Y, Bai L: Prognostic value of circulating inflammatory factors in nonsmall cell lung cancer: A systematic review and meta-analysis. Cancer Biomark 2014;14:469-481.

6 Cam C, Karagoz B, Muftuoglu T, Bigi O, Emirzeoglu L, Celik S, Ozgun A, Tuncel T, Top C: The inflammatory cytokine interleukin-23 is elevated in lung cancer, particularly small cell type. Contemp Oncol (Pozn) 2016;20:215-219.

7 He Z, Huang C, Lin G, Ye Y: Sirna-induced traf6 knockdown promotes the apoptosis and inhibits the invasion of human lung cancer spc-a1 cells. Oncol Rep 2016;35:1933-1940.

-8 Zhang J, Liang Y, Lin Y, Liu Y, YouYou, Yin W: Ire1alpha-traf2-ask1 pathway is involved in cstmpinduced apoptosis and er stress in human non-small cell lung cancer a549 cells. Biomed Pharmacother 2016;82:281-289.

9 Kanamori M, Suzuki H, Saito R, Muramatsu M, Hayashizaki Y: T2bp, a novel traf2 binding protein, can activate nf-kappab and ap-1 without tnf stimulation. Biochem Biophys Res Commun 2002;290:1108-1113.

10 Takatsuna H, Kato H, Gohda J, Akiyama T, Moriya A, Okamoto Y, Yamagata Y, Otsuka M, Umezawa K, Semba $\mathrm{K}$, Inoue J: Identification of tifa as an adapter protein that links tumor necrosis factor receptor-associated factor 6 (traf6) to interleukin-1 (il-1) receptor-associated kinase-1 (irak-1) in il-1 receptor signaling. J Biol Chem 2003;278:12144-12150.

11 Ea CK, Sun L, Inoue J, Chen ZJ: Tifa activates ikappab kinase (ikk) by promoting oligomerization and ubiquitination of traf6 Proc Natl Acad Sci U S A 2004;101:15318-15323.

12 Vlahopoulos SA, Cen O, Hengen N, Agan J, Moschovi M, Critselis E, Adamaki M, Bacopoulou F, Copland JA, Boldogh I, Karin M, Chrousos GP: Dynamic aberrant nf-kappab spurs tumorigenesis: A new model 


\section{Cellular Physiology Cell Physiol Biochem 2018;47:2097-2108 \begin{tabular}{l|l} 
DOI: 10.1159/000491478 & $\begin{array}{l}\text { O 2018 The Author(s). Published by S. Karger AG, Basel } \\
\text { www.karger.com/cpb }\end{array}$
\end{tabular} \\ Men et al.: TIFA in Lung Adenocarcinoma}

encompassing the microenvironment. Cytokine Growth Factor Rev 2015;26:389-403.

13 Escarcega RO, Fuentes-Alexandro S, Garcia-Carrasco M, Gatica A, Zamora A: The transcription factor nuclear factor-kappa b and cancer. Clin Oncol (R Coll Radiol) 2007;19:154-161.

14 Shen W, Chang A, Wang J, Zhou W, Gao R, Li J, Xu Y, Luo X, Xiang R, Luo N, Stupack DG: Tifa, an inflammatory signaling adaptor, is tumor suppressive for liver cancer. Oncogenesis 2015;4:e173.

15 Wei TW, Wu PY, Wu TJ, Hou HA, Chou WC, Teng CJ, Lin CR, Chen JM, Lin TY, Su HC, Huang CF, Yu CR, Hsu SL, Tien HF, Tsai MD: Aurora a and nf-kappab survival pathway drive chemoresistance in acute myeloid leukemia via the traf-interacting protein tifa. Cancer Res 2017;77:494-508.

16 Ferlay J, Soerjomataram I, Dikshit R, Eser S, Mathers C, Rebelo M, Parkin DM, Forman D, Bray F: Cancer incidence and mortality worldwide: Sources, methods and major patterns in globocan 2012 Int J Cancer 2015;136:E359-386.

17 Zou XN, Wan X, Dai Z, Yang GH: Epidemiological characteristics of cancer in elderly chinese. ISRN Oncol 2012;2012:381849.

18 Rothe M, Wong SC, Henzel WJ, Goeddel DV: A novel family of putative signal transducers associated with the cytoplasmic domain of the 75 kda tumor necrosis factor receptor. Cell 1994;78:681-692.

19 Cao Z, Xiong J, Takeuchi M, Kurama T, Goeddel DV: Traf6 is a signal transducer for interleukin-1. Nature 1996;383:443-446.

20 Ishida T, Mizushima S, Azuma S, Kobayashi N, Tojo T, Suzuki K, Aizawa S, Watanabe T, Mosialos G, Kieff E, Yamamoto T, Inoue J: Identification of traf6, a novel tumor necrosis factor receptor-associated factor protein that mediates signaling from an amino-terminal domain of the cd40 cytoplasmic region. J Biol Chem 1996;271:28745-28748.

21 Zhang XL, Dang YW, Li P, Rong MH, Hou XX, Luo DZ, Chen G: Expression of tumor necrosis factor receptorassociated factor 6 in lung cancer tissues. Asian Pac J Cancer Prev 2014;15:10591-10596.

-22 Minoda Y, Saeki K, Aki D, Takaki H, Sanada T, Koga K, Kobayashi T, Takaesu G, Yoshimura A: A novel zinc finger protein, zcchc11, interacts with tifa and modulates tlr signaling. Biochem Biophys Res Commun 2006;344:1023-1030.

23 Hayden MS, Ghosh S: Nf-kappab, the first quarter-century: Remarkable progress and outstanding questions. Genes Dev 2012;26:203-234.

24 Hoesel B, Schmid JA: The complexity of nf-kappab signaling in inflammation and cancer. Mol Cancer 2013;12:86.

25 Tergaonkar V, Pando M, Vafa O, Wahl G, Verma I: P53 stabilization is decreased upon nfkappab activation: A role for nfkappab in acquisition of resistance to chemotherapy. Cancer Cell 2002;1:493-503.

26 Mehta SV, Shukla SN, Vora HH: Overexpression of bcl2 protein predicts chemoresistance in acute myeloid leukemia: Its correlation with flt3 Neoplasma 2013;60:666-675.

27 Ding N, Zhang Y, Loughran PA, Wang Q, Tsung A, Billiar TR: Tifa upregulation after hypoxia-reoxygenation is tlr4- and myd88-dependent and associated with hmgb1 upregulation and release. Free Radic Biol Med 2013;63:361-367. 